\title{
Crossover Redefreiheit
}

\section{Ein Beitrag im Rahmen des Ri:Call for Papers: Redefreiheit und Louis D. Brandeis}

\author{
B Y U N G J IN PARK *
}

I.

Manchmal gibt es bei TV-Serien oder auch Filmen ein sogenanntes "Crossover". Bei einem Serien-Crossover treten mehrere Figuren aus voneinander unabhängigen Serien in einer gemeinsamen Episode auf. Doch nicht nur dort, sondern auch im realen Leben gibt es Crossover unterschiedlicher Epochen: So z.B. der Jahre 1927 und 2020 - Washington D.C. und Hannover.

\section{November 2020, Hannover, vor dem Opernhaus:}

Auf der Bühne steht Jana aus Kassel, 22 Jahre alt. Auf der Querdenker-Demonstration sagt sie unter anderem den folgenden Satz:

\begin{abstract}
"Ich fühle mich wie Sophie Scholl, da ich seit Monaten hier aktiv im Widerstand bin, Reden halte, auf Demos gehe, Flyer verteile und auch seit gestern Versammlungen anmelde."
\end{abstract}

Als sie weiter ausführt und unter anderem bekräftigt, sich weiter "für die Freiheit" einzusetzen, schreitet ein Ordner heran, wirft ihr die Verharmlosung des Holocaust vor und kündigt an, seine Ordner-Tätigkeit "für so einen Schwachsinn" nicht mehr auszuüben.

Jana bricht in Tränen aus, wirft das Mikrofon weg und verlässt die Bühne.²

\section{Mai 1927, Washington D.C., Supreme Court:}

Das Urteil gegen Charlotte Anita Whitney auf Basis des „1919 California Criminal Syndicalism Act" wegen ihrer Tätigkeit zur Gründung und Unterstützung der Kommunistischen
Arbeiterpartei Amerikas, welcher vorgeworfen wurde, den gewaltsamen Sturz der Regierung zu propagieren und zu lehren, wird vom Obersten Gerichtshof der Vereinigten Staaten bestätigt.

Louis D. Brandeis, der erste jüdische Richter am Obersten Gerichtshof der Vereinigten Staaten, legte zu der vorgenannten Entscheidung sein Sondervotum vor. Dort führte er insbesondere aus:

"If there be time to expose through discussion the falsehood and fallacies, to avert the evil by the process of education, the remedy to be applied is more speech, not enforced silence. Only an emergency can justify repression."

Charlotte Anita Whitney wurde später begnadigt. Der Begnadigung lag das Sondervotum des Richters Brandeis zugrunde.

II.

In Deutschland ist die Redefreiheit Teil der Meinungsfreiheit in Art. 5 Abs. 1 GG. Danach hat jeder das Recht, seine Meinung in Wort, Schrift und Bild frei zu äußern und zu verbreiten. Die Meinungsfreiheit findet ihre Grenzen - "Schranken" - in Art. 5 Abs. 2 GG: "Diese Rechte finden ihre Schranken in den Vorschriften der allgemeinen Gesetze, den gesetzlichen Bestimmungen zum Schutze der Jugend und in dem Recht der persönlichen Ehre." Die dort genannten allgemeinen Gesetze sollen, sofern sie die Meinungsfreiheit beschränken, ihrerseits im Lichte der Meinungsfreiheit ausgelegt werden, d.h. der Einschränkung der Meinungsfreiheit werden ebenfalls Grenzen - "Schranken-Schranken" - auferlegt (die "Wechselwirkungslehre" des Bundesverfassungsgerichts).

\footnotetext{
* Syndikusrechtsanwalt bei n-art-m GmbH in Karben und Promotionsstudent an der Julius-Maximilians-Universität in Würzburg

1 Video der "Rede" aufrufbar z.B. über WDR Aktuell YouTube unter: https://www.youtube.com/watch?v=gMuNRcBXe9c (zuletzt abgerufen am 16.11.2021).

2 Video der "Rede" aufrufbar z.B. über WDR Aktuell YouTube unter: https://www.youtube.com/watch?v=gMuNRcBXe9c (zuletzt abgerufen am 16.11.2021).

3 Whitney v. California, 274 U.S. 357 (1927), Volltext abrufbar unter: https://caselaw.findlaw.com/us-supreme-court/274/357.html (zuletzt abgerufen am 16.11.2021).
} 
Die Redefreiheit ("freedom of speech") ist in den USA im 1. Zusatzartikel der Verfassung der Vereinigten Staaten von Amerika verankert. ${ }^{4}$ Der Kongress darf demgemäß kein Gesetz erlassen, das die Redefreiheit einschränkt. Die größte Verteidigung der Redefreiheit in der US-amerikanischen Geschichte erfolgte im oben genannten Verfahren Whitney v. California im Jahr 1927 durch Louis D. Brandeis, einen der obersten Richter der Vereinigten Staaten von Amerika.

Brandeis führte in seinem Sondervotum zunächst aus, dass die Redefreiheit grundsätzlich eingeschränkt werden kann:

"The right of free speech, the right to teach and the right of assembly are, of course, fundamental rights. These may not be denied or abridged. But, although the rights of free speech and assembly are fundamental, they are not in their nature absolute. Their exercise is subject to restriction, if the particular restriction proposed is required in order to protect the State from destruction or from serious injury, political, economic or moral." ${ }^{\text {s }}$

Bezüglich der Einschränkung der Redefreiheit entwickelte zu dieser Zeit Oliver Wendell Homes Jr., ebenfalls Richter am Obersten Gerichtshof der Vereinigten Staaten, den sogenannten "clear and present danger" Test. In der Schenck V. United States Entscheidung aus dem Jahr 1919 führte er dazu wie folgt aus:

"The question in every case is whether the words used are used in such circumstances and are of such a nature as to create a clear and present danger that they will bring about the substantive evils that Congress has a right to prevent." 6

Demnach war die Redefreiheit dann einzuschränken, wenn durch die Ausübung dieses Rechts eine "eindeutige und gegenwärtige Gefahr" geschaffen würde. Wann eine Gefahr für die Gemeinschaft jedoch eindeutig und gegenwärtig und damit die Einschränkung der Redefreiheit gerechtfertigt ist, war auf höchstrichterlicher Ebene noch nicht geklärt, worauf Brandeis in seinem Sondervotum hinwies:

"This Court has not yet fixed the standard by which to determine when a danger shall be deemed clear; how remote the danger may be and yet be deemed present; and what degree of evil shall be deemed sufficiently substantial to justify resort to abridgement of free speech and assembly as the means of protection." 7
Brandeis entwickelte den "clear and present danger" Test sodann weiter und sprach von der Voraussetzung einer nicht nur "gegenwärtigen", sondern „imminenten" Gefahr:

"Fear of serious injury cannot alone justify suppression of free speech and assembly. Men feared witches and burnt women. It is the function of speech to free men from the bondage of irrational fears. To justify suppression of free speech there must be reasonable ground to fear that serious evil will result if free speech is practiced. There must be reasonable ground to believe that the danger apprehended is imminent." 8

Für Brandeis musste die Möglichkeit, die Regierungspolitik kritisieren zu können ohne Repressalien befürchten zu müssen, gewährleistet sein. Er machte deutlich, dass für die Durchsetzung der Einschränkung der Redefreiheit hohe Hürden zu überwinden seien. Solange nicht durch die Ausübung der Redefreiheit unmittelbar und imminente Gefahr für die Gemeinschaft bzw. für den Staat entstehe (um sich eine solche imminente Gefahr vorzustellen, dürfte beispielsweise der Blick auf den Sturm auf das Kapitol in Washington im Januar 2021 hilfreich sein), müsse die Lösung lauten: "more speech, not enforced silence". Die Redefreiheit war für Brandeis für den demokratischen Prozess unabdingbar; unpopuläre Meinungen müssen geäußert und über diese müsse diskutiert werden können. Staatliche Repression würden Hass erzeugen, und Hass bedrohe die gesellschaftliche Stabilität. Brandeis forderte die Amerikaner dazu auf, die Redefreiheit aktiv zu verteidigen und Gesetze, die die Redefreiheit ohne ausreichende Gründe ("clear and imminent danger") einschränken, anzufechten.

Brandeis' Sondervotum wird zu Recht als die größte Verteidigung der Redefreiheit in der Geschichte der Vereinigten Staaten von Amerika bezeichnet.

\section{III.}

Fast forward ins Deutschland des 21. Jahrhunderts. Im Gegensatz zu 1927 befinden wir uns weder in einem Krieg noch haben wir einen solchen hinter oder vor uns. Stattdessen wütet seit fast zwei Jahren die COVID-19-Pandemie. In diesem Zusammenhang entstand die Querdenken-Bewegung. Mit Beginn der Schutzmaßnahmen von Bund und Ländern zur Eindämmung der Pandemie begannen auch die Proteste mit der Behauptung, damit würde das Grundgesetz gegen Staatsübergriffe verteidigt. Seither fanden diverse Demonstrationen in verschiedenen Städten statt - die Demonstra-

\footnotetext{
4 https://usa.usembassy.de/etexts/gov/gov-constitutiond.pdf (zuletzt abgerufen am am 16.11.2021).

5 Whitney v. California, 274 U.S. 357 (1927), Volltext abrufbar unter: https://caselaw.findlaw.com/us-supreme-court/274/357.html (zuletzt abgerufen am 16.11.2021).

6 Schenck v. United States, 249 U.S. 47 (1919), Volltext aufrufbar unter: https://caselaw.findlaw.com/us-supreme-court/249/47.html (zuletzt abgerufen am am 16.11.2021).

7 Whitney v. California, 274 U.S. 357 (1927), Volltext abrufbar unter: https://caselaw.findlaw.com/us-supreme-court/274/357.html (zuletzt abgerufen am 16.11.2021).

8 Whitney v. California, 274 U.S. 357 (1927), Volltext abrufbar unter: https://caselaw.findlaw.com/us-supreme-court/274/357.html (zuletzt abgerufen am 16.11.2021).
} 
tion in Hannover am 21. November 2020 sollte dabei "dank" Jana aus Kassel besondere Aufmerksamkeit erhalten.

Auf die inhaltliche Bewertung des Vergleichs mit der Widerstandskämpferin Sophie Scholl muss nicht näher eingegangen werden; Jana aus Kassel hat im Gegensatz zu Sophie Scholl ihrerzeit keine Repressalien zu befürchten. Stattdessen ging das Video von ihrem Auftritt viral (das Wortspiel ist an dieser Stelle beabsichtigt). Überwiegend gab es neben scharfer Kritik vor allem Häme für Jana. Die Medien behandelten das Thema sachlich; neben der inhaltlichen Bewertung des Sophie-Scholl-Vergleichs wurde beispielsweise hingewiesen auf die Wichtigkeit der Einordnung, in welchem Zusammenhang die Aussagen getätigt wurden und wie Querdenker systematisch die Geschichte umdeuten. ${ }^{9}$ Analysiert wurde auch der gegen die Demonstrantin entstandene Shitstorm im Netz. ${ }^{10}$

Besonders erwähnenswert ist vor allem der Kommentar von Wilm Hüffer im SWR2 vom 22. November 2020.11 Hüffer kommentierte den Auftritt des Ordners wie folgt:

"Ein klares, hartes Widerwort - schon das war zu viel für sie. Dabei sind solche Widerworte jetzt unerlässlich." 12

Noch wichtiger sind Hüffers Ausführungen am Ende seines Kommentars:

\begin{abstract}
"Über Nacht ist aus Jana aus Kassel eine neue Hassfigur geworden. In sozialen Netzwerken wie Facebook und Twitterhageltes Beschimpfungen und Verwünschungen.

Das ist nachvollziehbar. Doch darin besteht auch eine Gefahr. Wütende Reaktionen werden die sogenannten Querdenker nicht zur Vernunft bringen. Ganz im Gegenteil. Wut und Hass werden diese Menschen in ihrer absurden Selbstwahrnehmung nur bestätigen. Werden sie in ihrer selbstgerechten Überzeugung bestärken, sie lebten in einer Meinungsdiktatur, in der sie nicht mehr offen reden dürften.
\end{abstract}

Genau hier beginnen die gefährlichen Mechanismen, die unsere Demokratien bedrohen: Hass erzeugt Gegenhass - und am Ende die Unfähigkeit, sich auseinander- zusetzen. Deshalb: klare Kante gegen alle, die sich wie Sophie Scholl zu fühlen glauben. Aber bitte mit Argumenten. Ohne Schaum vor dem Mund. Ohne Wut, ohne Hass. Denn beides hilft am Ende nur den Demagogen." ${ }^{13}$

Auch wenn es hierbei nicht um staatliche Repression geht: Hass erzeugt Gegenhass und bedroht am Ende die gesellschaftliche Stabilität. Es ist das berühmte "Aushaltenmüssen": Unpopuläre Meinungen müssen geäußert werden können und über diese muss diskutiert werden können. Der Diskurs - der Austausch von Meinungen und Ideen, die Reibung und die dadurch erzeugte Energie oder Schlussfolgerung - ist die zentrale Grundlage unserer Demokratie und die Redefreiheit als Teil der Meinungsfreiheit daher ein unverzichtbares, hohes Gut. Der Staat hat dafür Sorge zu tragen, dass die Bürger ihre Meinungsfreiheit - innerhalb der geltenden Schranken - ausüben können. Im Zweifel bedeutet dies, dass selbst Ansichten und Vorstellungen darüber, dass in Deutschland im 21. Jahrhundert eine Meinungsdiktatur herrsche, öffentlich bekundet werden und auf offener, durch Ordner geschützter Bühne behauptet werden kann, man dürfe nicht mehr offen reden.

\section{IV.}

Opernplatz in Hannover als der von Holmes und Brandeis ("free trade in ideas within the competition of the market"14) beschriebene Marktplatz der Ideen (" marketplace of ideas ${ }^{\prime 15}$ ):

Brandeis' Ansichten in Gestalt des Ordners der Querdenken-Veranstaltung, der einerseits die Redefreiheit schützt, andererseits Janas Rede herausfordert und damit die Diskussion darüber entfacht.

Eine Gesellschaft, in welcher unpopuläre Meinungen kundgetan werden können, ohne dass die Kundgebenden staatliche Konsequenzen fürchten müssen, in welcher über diese Meinung diskutiert werden kann, in welcher die Rede- bzw. Meinungsfreiheit gewährleistet ist und die hohe, wichtige Stellung einnimmt, die Brandeis im Jahr 1927 in seinem Sondervotum skizzierte.

Welch ein gelungenes Crossover Redefreiheit.

\footnotetext{
9 Deutschlandfunk Nova, Interview mit Pia Lamberty vom 24.11.2020, Sozialpsychologin an der Universität Mainz, aufrufbar unter: https://www.deutschlandfunknova.de/beitrag/janaauskassel-es-ist-zu-leicht-sich-ueber-jana-nur-lustig-zu-machen (zuletzt abgerufen am 16.11.2021).

10 Ruhdorfer in Krautreporter vom 26.11.2020, abrufbar unter: https://krautreporter.de/3573-jana-aus-kassel-hat-uns-den-moment-beschert-den-wir-uns-seit-monaten-wunschen (zuletzt abgerufen am 16.11.2021).

11 Hüffer, SWR2, Kommentar vom 22.11.2020, abrufbar unter: https://www.swr.de/swr2/leben-und-gesellschaft/gefahr-fuer-die-demokratie-jana-aus-kassel-fuehlt-sich-wie-sophiescholl-100.html (zuletzt abgerufen am 16.11.2021).

12 Hüffer, SWR2, Kommentar vom 22.11.2020, abrufbar unter: https://www.swr.de/swr2/leben-und-gesellschaft/gefahr-fuer-die-demokratie-jana-aus-kassel-fuehlt-sich-wie-sophiescholl-100.html (zuletzt abgerufen am 16.11.2021).

13 Hüffer, SWR2, Kommentar vom 22.11.2020, abrufbar unter: https://www.swr.de/swr2/leben-und-gesellschaft/gefahr-fuer-die-demokratie-jana-aus-kassel-fuehlt-sich-wie-sophiescholl-100.html (zuletzt abgerufen am 16.11.2021).

14 Abrams v. United States, 250 U.S. 616 (1919), Volltext abrufbar unter https://supreme.justia.com/cases/federal/us/250/616/\#619 (zuletzt abgerufen am 16.11.2021).

15 So zum ersten Mal bezeichnet von Richter William O. Douglas in dessen Sondervotum zur Entscheidung United States v. Rumely, 345 U.S. 41,56 (1953).
} 